\title{
A high blood endocan profile during COVID-19 distinguishes moderate from severe acute respiratory distress syndrome
}

\author{
Tiffany Pascreau ${ }^{1,2^{*}}$ (i), Colas Tcherakian ${ }^{3}$, Benjamin Zuber ${ }^{4}$, Eric Farfour ${ }^{1}$, Marc Vasse ${ }^{1,2}$ and Philippe Lassalle
}

Endocan is expressed by the lung endothelial cells and upregulated by pro-inflammatory conditions. Because elevated pro-inflammatory cytokines are hallmarks of severe SARS-CoV-2 infection [1], and that the lung is the organ preferentially affected, we measured endocan in patients with COVID-19 pneumonia. This retrospective study was conducted between March 12 and April 20, 2020, and approved by the local ethics committee of the Foch Hospital (20-07-15). Seventy-four patients with COVID-19 pneumonia confirmed by RT-PCR were enrolled. According to the Berlin definition of acute respiratory distress syndrome (ARDS), patients were categorized into non-ARDS, mild/moderate ARDS, and severe ARDS. At the admission complete blood count, biochemical and coagulation parameters were measured. Endocan and cathepsin G-cleaved endocan (p14) concentrations were measured on baseline and during the hospitalization. Plasmatic endocan cleavage ratio (ECR) was calculated as plasma p14/(endocan + p14) ratio. Healthy hospital workers served as group of control. Baseline characteristics and clinical outcomes are summarized in Table 1 . Patients with ARDS had significantly higher CRP (176 mg/L [IQR: 133-270] vs $141 \mathrm{mg} / \mathrm{L}$ [IQR: $88-187$ ], $p=0.0122)$, and higher d-dimers $(1.843 \mathrm{mg} / \mathrm{L}$ [IQR: 0.579-7.134] vs $0.771 \mathrm{mg} / \mathrm{L}$ [IQR: 0.535-1.374], $p=0.0472$ ), had greater lung parenchyma involvement assessed by the CT score $(4[4,5]$ vs $3[2-4], p=0.0016)$

*Correspondence: t.pascreau@hopital-foch.com

1 Department of CLINICAL Biology, Foch Hospital, 40 rue Worth, 92150 Suresnes, France

Full list of author information is available at the end of the article and stay hospitalized for longer than non-ARDS group (25 days [IQR: $14-38$ ] vs 12 days [IQR: $7-17], p<0.0001$ ). The mortality rate was also higher in patients with ARDS than non-ARDS group ( $43 \%$ vs $5 \%, p<0.0001$ ) (Table 1 ). At admission, endocan levels measured in 59/74 (84\%) patients were significantly increased in patients with COVID-19 compared to controls $(3.4 \mathrm{ng} / \mathrm{mL}$ [IQR: $1.8-7.5$ ] vs $1.6 \mathrm{ng} / \mathrm{mL}$ [IQR: 1.0-2.1], respectively, $p=0.0031$ ) (Fig. 1a). There was no significant difference between patients who developed ARDS and those who have not $(3.7[2.8-9.6] \mathrm{ng} / \mathrm{mL}$ vs $3.2[1.5-5.7] \mathrm{ng} / \mathrm{mL}$, respectively, $p=0.2231$ ) (Fig. 1b). Endocan was negatively correlated with the platelets (Spearman's correlation coefficient $r=-0.3681, p=0.0041$ ). When plasma samples were available in patients with ARDS, endocan concentrations were measured during the hospitalization. Patients with mild/moderate ARDS had a significant increase in endocan levels at days 3-4 $(p=0.0084)$ and days 5-6 $(p=0.0107)$ compared to those measured at days 1-2 (Fig. 1c). No increase was observed in patients with severe ARDS (Fig. 1c). This discrepancy was not due to an increase in cleavage of endocan since the ECR remained similar whatever the severity of ARDS or the hospitalization day (not shown). ECR at admission was positively correlated with the Von Willebrand antigen ( $r=0.3047, p=0.0418)$. ARDS was already present at admission in 15/37 (40\%). Twelve (32\%) patients developed ARDS within $48 \mathrm{~h}$, whereas 10 (27\%) patients developed ARDS between the 3rd and 7th day of hospitalization. After exclusion of patients with ARDS within the first $48 \mathrm{~h}$, the calculated AUC of endocan was $0.7235(p=0.1104)$. Several biomarkers had already been original author(s) and the source, provide a link to the Creative Commons licence, and indicate if changes were made. The images or other third party material in this article are included in the article's Creative Commons licence, unless indicated otherwise in a credit line to the material. If material is not included in the article's Creative Commons licence and your intended use is not permitted by statutory regulation or exceeds the permitted use, you will need to obtain permission directly from the copyright holder. To view a copy of this licence, visit http://creativecommons.org/licenses/by/4.0/. The Creative Commons Public Domain Dedication waiver (http://creativeco mmons.org/publicdomain/zero/1.0/) applies to the data made available in this article, unless otherwise stated in a credit line to the data. 
Table 1 Baseline characteristics, biological and radiological findings at the admission and clinical outcomes of the patients

\begin{tabular}{|c|c|c|c|c|c|c|c|}
\hline & All $(n=74)$ & $\begin{array}{l}\text { Non-ARDS } \\
(n=37)\end{array}$ & ARDS $(n=37)$ & $\begin{array}{l}\text { ARDS vs non- } \\
\text { ARDS }\end{array}$ & $\begin{array}{l}\text { Mild or } \\
\text { moderate ARDS } \\
(n=12)\end{array}$ & $\begin{array}{l}\text { Severe ARDS } \\
(n=25)\end{array}$ & $\begin{array}{l}\text { Non-ARDS vs } \\
\text { mild/moderate } \\
\text { vs severe ARDS }\end{array}$ \\
\hline Male sex, $n(\%)$ & $\begin{array}{l}59(80 \%) \\
(n=74)\end{array}$ & $\begin{array}{l}30(81 \%) \\
(n=37)\end{array}$ & $29(78 \%)$ & $>0.9999$ & $9(75 \%)$ & $20(80 \%)$ & 0.9008 \\
\hline $\begin{array}{l}\text { Age, median [IQR] } \\
\text { (years) }\end{array}$ & $\begin{array}{l}64[55-71] \\
(n=74)\end{array}$ & $\begin{array}{l}65[51-73] \\
(n=37)\end{array}$ & $62[56-70]$ & 0.8486 & $63[56-76]$ & $62[55-68]$ & 0.6131 \\
\hline BMI (kg/m2) & $\begin{array}{l}26.9[24.0-31.0] \\
(n=53)\end{array}$ & $\begin{array}{l}26.5[23.7-30.6] \\
(n=30)\end{array}$ & $\begin{array}{l}27.3[24.2-31.0] \\
(n=23)\end{array}$ & 0.4355 & $\begin{array}{l}24.2[23.4-32.7] \\
(n=7)\end{array}$ & $\begin{array}{l}27.7[25.4-30.6] \\
(n=16)\end{array}$ & 0.5424 \\
\hline \multicolumn{8}{|l|}{ Comorbidities } \\
\hline $\begin{array}{l}\text { Hypertension } \\
n(\%)\end{array}$ & $\begin{array}{l}36(49 \%) \\
(n=74)\end{array}$ & $19(63 \%)$ & $17(46 \%)$ & 0.8163 & $5(42 \%)$ & $12(48 \%)$ & 0.8408 \\
\hline Diabetes $n(\%)$ & $\begin{array}{l}31(42 \%) \\
(n=74)\end{array}$ & $14(47 \%)$ & $17(46 \%)$ & 0.6378 & $7(58 \%)$ & $10(40 \%)$ & 0.4450 \\
\hline \multicolumn{8}{|c|}{ Biological and radiological parameters at admission } \\
\hline $\begin{array}{l}\text { CRP (mg/L) } \\
N<5 \mathrm{mg} / \mathrm{L}\end{array}$ & $\begin{array}{l}155[109-217] \\
(n=70)\end{array}$ & $\begin{array}{l}141[88-187] \\
(n=34)\end{array}$ & $\begin{array}{l}176[133-270] \\
(n=36)\end{array}$ & 0.0122 & $\begin{array}{l}178[125-301] \\
(n=12)\end{array}$ & $\begin{array}{l}175[133-267] \\
(n=24)\end{array}$ & 0.0447 \\
\hline $\begin{array}{l}\text { Creatinine } \\
(\mu \mathrm{mol} / \mathrm{L}) \\
\text { N49-90 (F); } \\
64-104(\mathrm{M}) \\
\mu \mathrm{mol} / \mathrm{L}\end{array}$ & $\begin{array}{l}95[70-131] \\
(n=74)\end{array}$ & $\begin{array}{l}100[74-132] \\
(n=37)\end{array}$ & $\begin{array}{l}93[67-141] \\
(n=37)\end{array}$ & 0.4794 & $\begin{array}{l}98[65-127] \\
(n=12)\end{array}$ & $\begin{array}{l}93[68-165] \\
(n=25)\end{array}$ & 0.7714 \\
\hline $\begin{array}{l}\text { AST (UI/L) } \\
\text { N 5-34 UI/L }\end{array}$ & $\begin{array}{l}63[42-88] \\
(n=70)\end{array}$ & $\begin{array}{l}57[40-84] \\
(n=36)\end{array}$ & $\begin{array}{l}72[43-98] \\
(n=34)\end{array}$ & 0.1550 & $\begin{array}{l}65[39-111] \\
(n=10)\end{array}$ & $\begin{array}{l}77[45-90] \\
(n=24)\end{array}$ & 0.3262 \\
\hline $\begin{array}{l}\text { ALT (UI/L) } \\
N<55 \mathrm{UI} / \mathrm{L}\end{array}$ & $\begin{array}{l}32[21-52] \\
(n=70)\end{array}$ & $\begin{array}{l}28[21-50] \\
(n=36)\end{array}$ & $\begin{array}{l}34[23-53] \\
(n=34)\end{array}$ & 0.4846 & $\begin{array}{l}40[12-53] \\
(n=10)\end{array}$ & $\begin{array}{l}34[23-60] \\
(n=24)\end{array}$ & 0.8455 \\
\hline $\begin{array}{l}\text { Ferritin }(\mu \mathrm{g} / \mathrm{L}) \\
N \text { 5-204 (F); } \\
22-275(\mathrm{M}) \mu \mathrm{g} / \mathrm{L}\end{array}$ & $\begin{array}{l}1522[770-2688] \\
(n=51)\end{array}$ & $\begin{array}{l}1183[574-2291] \\
(n=24)\end{array}$ & $\begin{array}{l}1602[1068-2704] \\
(n=27)\end{array}$ & 0.1773 & $\begin{array}{l}1252[599-2531] \\
(n=8)\end{array}$ & $\begin{array}{l}2064[1104-4130] \\
(n=19)\end{array}$ & 0.1966 \\
\hline $\begin{array}{l}\text { Platelets }\left(10^{9} / \mathrm{L}\right) \\
\text { N 150-450 109/L }\end{array}$ & $\begin{array}{l}189[143-244] \\
(n=73)\end{array}$ & $\begin{array}{l}186[143-244] \\
(n=37)\end{array}$ & $\begin{array}{l}191[142-252] \\
(n=36)\end{array}$ & 0.8242 & $\begin{array}{l}205[152-268] \\
(n=12)\end{array}$ & $\begin{array}{l}182[130-229] \\
(n=24)\end{array}$ & 0.6761 \\
\hline $\begin{array}{l}\text { PNN }\left(10^{9} / \mathrm{L}\right) \\
N 1.5-7.510^{9} / \mathrm{L}\end{array}$ & $\begin{array}{l}6.4[4.3-8.8] \\
(n=73)\end{array}$ & $\begin{array}{l}6.1[4.1-7.6] \\
(n=37)\end{array}$ & $\begin{array}{l}7.0[4.5-9.0] \\
(n=36)\end{array}$ & 0.0750 & $\begin{array}{l}8.1[4.4-9.0] \\
(n=12)\end{array}$ & $\begin{array}{l}7.0[4.5-9.5] \\
(n=24)\end{array}$ & 0.1857 \\
\hline $\begin{array}{l}\text { VWF antigen (\%) } \\
\text { N 50-150\% }\end{array}$ & $\begin{array}{l}429[331-498] \\
(n=64)\end{array}$ & $\begin{array}{l}347[299-509] \\
(n=35)\end{array}$ & $\begin{array}{l}449[368-494] \\
(n=29)\end{array}$ & 0.1648 & $\begin{array}{l}476[403-546] \\
(n=10)\end{array}$ & $\begin{array}{l}446[331-484] \\
(n=19)\end{array}$ & 0.2497 \\
\hline $\begin{array}{l}\text { Fibrinogen }(\mathrm{g} / \mathrm{L}) \\
N 1.5-3.5 \mathrm{~g} / \mathrm{L}\end{array}$ & $\begin{array}{l}6.10[4.15-6.82] \\
(n=52)\end{array}$ & $\begin{array}{l}5.14[4.03-6.46] \\
(n=28)\end{array}$ & $\begin{array}{l}6.32[4.37-7.13] \\
(n=24)\end{array}$ & 0.1056 & $\begin{array}{l}6.40[6.14-7.11] \\
(n=8)\end{array}$ & $\begin{array}{l}6.18[4.24-7.13] \\
(n=16)\end{array}$ & 0.2153 \\
\hline $\begin{array}{l}\text { D-dimers (mg/L) } \\
N<0.500 \mathrm{mg} / \mathrm{L}\end{array}$ & $\begin{array}{l}1.079[0.538-3.607] \\
(n=61)\end{array}$ & $\begin{array}{l}0.771[0.535- \\
1.374] \\
(n=31)\end{array}$ & $\begin{array}{l}1.843[0.579- \\
7.134] \\
(n=30)\end{array}$ & 0.0472 & $\begin{array}{l}1.492[0.569- \\
3.228] \\
(n=10)\end{array}$ & $\begin{array}{l}1.843[0.586- \\
17.64] \\
(n=20)\end{array}$ & 0.1207 \\
\hline $\begin{array}{l}\text { Initial CT findings } \\
\text { (score) })^{*}\end{array}$ & $\begin{array}{l}4[3-4] \\
(n=39)\end{array}$ & $\begin{array}{l}3[2-4] \\
(n=22)\end{array}$ & $\begin{array}{l}4[4-5] \\
(n=17)\end{array}$ & 0.0016 & $\begin{array}{l}4[4-5] \\
(n=9)\end{array}$ & $\begin{array}{l}4[4-5] \\
(n=6)\end{array}$ & 0.0171 \\
\hline \multicolumn{8}{|l|}{ Clinical outcomes } \\
\hline $\begin{array}{l}\text { Time from illness } \\
\text { onset to hos- } \\
\text { pital admission } \\
\text { (days) }\end{array}$ & $\begin{array}{l}8[6-12] \\
(n=69)\end{array}$ & $\begin{array}{l}9[7-12] \\
(n=34)\end{array}$ & $\begin{array}{l}8[5-12] \\
(n=37)\end{array}$ & 0.7217 & $\begin{array}{l}8[4-10] \\
(n=11)\end{array}$ & $\begin{array}{l}8[5-12] \\
(n=24)\end{array}$ & 0.5818 \\
\hline $\begin{array}{l}\text { Time from illness } \\
\text { onset to occur- } \\
\text { rence of ARDS }\end{array}$ & - & - & $\begin{array}{l}10[8-14] \\
(n=32)\end{array}$ & - & $\begin{array}{l}8[5-14] \\
(n=9)\end{array}$ & $\begin{array}{l}11[8-15] \\
(n=23)\end{array}$ & 0.1711 \\
\hline $\begin{array}{l}\text { Hospital stay } \\
\text { (days) }\end{array}$ & $\begin{array}{l}16[10-29] \\
(n=73)\end{array}$ & $\begin{array}{l}12[7-17] \\
(n=36)\end{array}$ & $\begin{array}{l}25[14-38] \\
(n=35)\end{array}$ & $<0.0001$ & $\begin{array}{l}23[14-30] \\
(n=12)\end{array}$ & $\begin{array}{l}27[14-41] \\
(n=25)\end{array}$ & 0.0004 \\
\hline Mortality n (\%) & $\begin{array}{l}18(24 \%) \\
(n=74)\end{array}$ & $\begin{array}{l}2(5 \%) \\
(n=37)\end{array}$ & $\begin{array}{l}16(43 \%) \\
(n=37)\end{array}$ & 0.0001 & $\begin{array}{l}3(25 \%) \\
(n=12)\end{array}$ & $13(52 \%)$ & 0.0002 \\
\hline
\end{tabular}

* Semiquantitative CT score was calculated based on the extent of lung parenchyma involvement (1: 0-10\%; 2: 11-25\%; 3: 26-50\%; 4: 51-75\%; 5: > 75\%)

In univariate analysis, we determined the differences in median using unpaired t test (Mann-Whitney $U$ test) for continuous variable and differences in proportions were determined using the Chi-square test or Fischer exact test, as appropriate. Concentrations of endocan were compared between groups (control, non-ARDS, mild/ moderate ARDS and severe ARDS) using the Kruskal-Wallis test followed by Dunn's posttest. Bold font indicates statistical significance

AST aspartate aminotransferase, ALT alanine aminotransferase, $P N N$ polynuclear neutrophils, VWF von Willebrand factor 


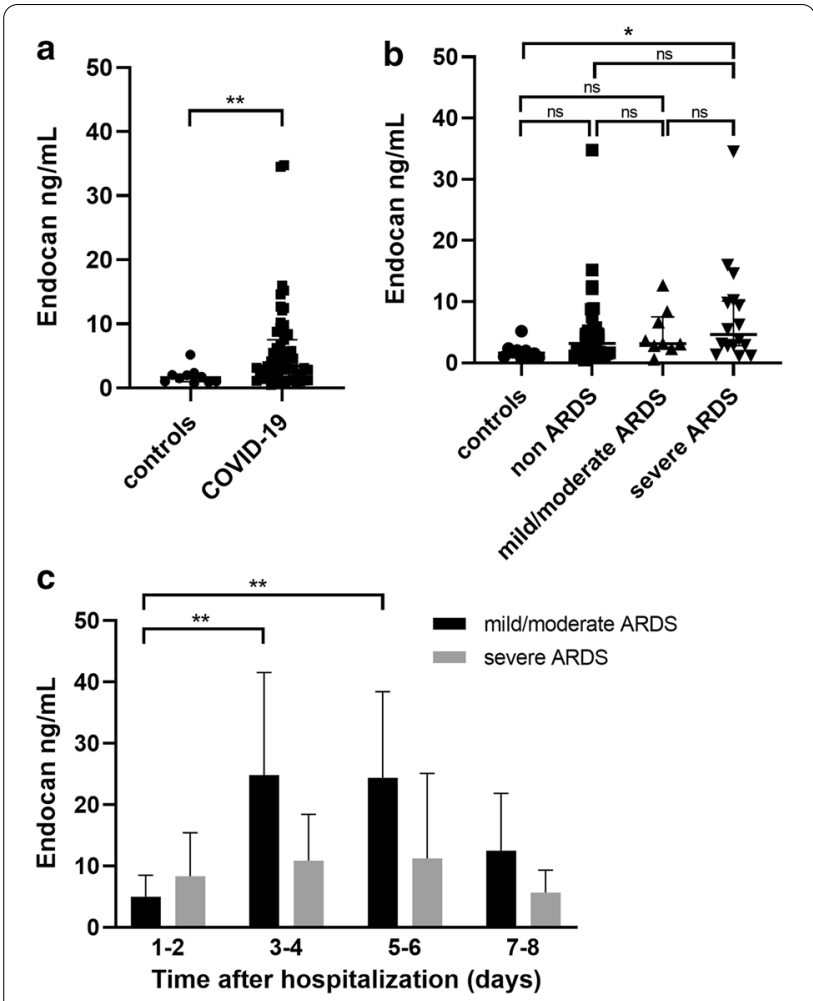

Fig. 1 Endocan concentrations in patients with COVID-19 at admission and during the hospitalization. a Endocan levels measured at the admission in patients with COVID-19. Data are presented as median and interquartile range. Patients with COVID-19 were compared to the control group using the Mann-Whitney test. ${ }^{*} p<0.05$. $\mathbf{b}$ Endocan levels measured at the admission in patients with COVID-19 categorized into non-ARDS, mild/moderate ARDS and severe ARDS. Data are presented as median and interquartile range. Results were expressed as mean and standard deviation. Intergroup comparison was made using the Kruskal-Wallis test. ${ }^{*} p<0.05$. c Endocan levels measured over the course of hospitalization in patients with COVID-19 and ARDS. Mild/moderate ARDS group $n=10$ (days $1-2$ ), $n=3$ (days $3-4$ ), $n=3$ (days $5-6$ ), $n=2$ (days 7-8); severe ARDS group $n=18$ (days 1-2), $n=12$ (days $3-4$ ), $n=13$ (days $5-6$ ), $n=9$ (days 7-8). Results are expressed as mean \pm standard deviation. Difference in endocan levels over the course of the hospitalization was calculated using the two-way ANOVA followed by Bonferroni's posttest. ${ }^{* *} p<0.01$

related to the ARDS $[2,3]$. In a series of 659 patients with COVID-19, lymphocyte count, creatine kinase, neutrophils/lymphocytes ratio, AST, lactate dehydrogenase, and CRP were all strongly related to the aggravation of ARDS [4]. Few data are available in the literature about endocan in COVID-19. One study observed that endocan levels at the admission were associated with poor clinical outcomes, but the occurrence of ARDS has not been studied [5]. In our series, endocan levels measured at admission could be predictive of ARDS after the 3rd day of hospitalization. During the hospitalization, we observed a lack of increase in endocan levels in patients with severe ARDS which is consistent with the finding that low endocan levels during sepsis may also predict ARDS worsening [6]. Further prospective studies are required to confirm these results on the role of endocan in prediction of ARDS in patients with COVID-19.

\section{Abbreviations}

ARDS: Acute respiratory distress syndrome; AST: Aspartate aminotransferase; AUC: Area under curve; COVID-19: Coronavirus disease 2019; CRP: C-reactive protein; CT: Computed tomography; ECR: Endocan cleaved ratio; IQR: Interquartile range.

\section{Acknowledgements}

Not applicable.

\section{Authors' contributions}

TP performed the endocan measurement. TP, PL analyzed the data. TP, BZ collected the data. All authors read and approved the final manuscript.

Funding

Not applicable.

\section{Availability of data and materials}

The datasets analyzed during the current study are available from the corresponding author on reasonable request.

\section{Declarations}

\section{Ethics approval}

The study was approved by the local ethics committee of the Foch Hospital (reference 20-07-15).

\section{Consent for publication}

Not applicable.

\section{Competing interests}

The authors declare that they have no competing interests.

\section{Author details}

${ }^{1}$ Department of CLINICAL Biology, Foch Hospital, 40 rue Worth, 92150 Suresnes, France. ${ }^{2}$ INSERM UMR_S 1176, University Paris-Sud, University Paris-Saclay, Le Kremlin-Bicêtre, France. ${ }^{3}$ Department of Pneumology, Foch Hospital, Suresnes, France. ${ }^{4}$ Department of Medical and Surgical Intensive Care Unit, Foch Hospital, Suresnes, France. ${ }^{5}$ Univ. Lille, CNRS, Inserm, CHU Lille, Institut Pasteur de Lille, U1019-UMR9017-CIIL-Centre d'Infection et d'Immunité de Lille, Equipe immunité pulmonaire, Lille, France. ${ }^{6}$ Biothelis, 59000 Lille, France.

Received: 21 April 2021 Accepted: 27 April 2021

Published online: 06 May 2021

\section{References}

1. Chen G, Wu D, Guo W, Cao Y, Huang D, Wang H, et al. Clinical and immunological features of severe and moderate coronavirus disease 2019. J Clin Invest. 2020;130(5):2620-9.

2. van der Zee P, Rietdijk W, Somhorst P, Endeman H, Gommers D. A systematic review of biomarkers multivariately associated with acute respiratory distress syndrome development and mortality. Crit Care. 2020;24(1):243.

3. Gaudet A, Parmentier E, De Freitas CN, Portier L, Dubucquoi S, Poissy J, et al. Decrease of the plasmatic endocan cleavage ratio is associated 
with the hyperinflammatory phenotype of acute respiratory distress syndrome. Crit Care. 2019;23(1):252.

4. Xu W, Sun N-N, Gao H-N, Chen Z-Y, Yang Y, Ju B, et al. Risk factors analysis of COVID-19 patients with ARDS and prediction based on machine learning. Sci Rep. 2021;11(1):2933.

5. Medetalibeyoglu A, Emet S, Kose M, Akpinar TS, Senkal N, Catma Y, et al. Serum endocan levels on admission are associated with worse clinical outcomes in COVID-19 patients: a pilot study. Angiology. 2021;72(2):187-93.
6. Gaudet A, Parmentier E, Dubucquoi S, Poissy J, Duburcq T, Lassalle P, et al. Low endocan levels are predictive of Acute Respiratory Distress Syndrome in severe sepsis and septic shock. J Crit Care. 2018:47:121-6.

\section{Publisher's Note}

Springer Nature remains neutral with regard to jurisdictional claims in published maps and institutional affiliations.
Ready to submit your research? Choose BMC and benefit from:

- fast, convenient online submission

- thorough peer review by experienced researchers in your field

- rapid publication on acceptance

- support for research data, including large and complex data types

- gold Open Access which fosters wider collaboration and increased citations

- maximum visibility for your research: over $100 \mathrm{M}$ website views per year

At BMC, research is always in progress.

Learn more biomedcentral.com/submissions 